\title{
Concentração de Rn-222 e filhos em águas provenientes de poços e emergências de água da região de Presidente Prudente: resultados preliminares
}

\author{
Ana Maria Araya Osório ${ }^{1,2}$, Claudinei Rodrigues de Aguiar ${ }^{3}$, Carlos Alberto Tello Saenz ${ }^{1}$, Luiz Augusto Stuani \\ Pereira $^{4}$ \\ ${ }^{1}$ Docente do Departamento de Física Química e Biologia da FCT UNESP, Presidente Prudente. ${ }^{2}$ Coordenadora do grupo de Pesquisa \\ DETRANES e o grupo de Ensino em Física Moderna. ${ }^{3}$ Docente da Universidade Tecnológica Federal do Paraná. ${ }^{4}$ Aluno do curso de \\ Graduação em Física da UNESP, Presidente Prudente, Bolsista CNPq.
}

\section{Resumo}

Neste trabalho apresentamos os resultados preliminares da atividade do Rn-222 e filhos em águas provenientes de poços e emergências de água da região de Presidente Prudente. Foram estudadas seis amostras; três amostras vindas de poços, duas amostras de emergências de água e uma amostra de água potável. Para a medida da atividade do Rn-222 e filhos, as amostras de água foram colocadas em recipientes plásticos hermeticamente fechados, nos quais detectores CR-39 foram expostos ao ar ambiental vindo da água. Para obter a densidade de traços de partículas $\alpha$, provenientes do decaimento do Rn-222 e filhos, utilizamos um microscópio óptico de luz transmitida. Os resultados mostram que a amostra $A-3$, que corresponde à água retirada de um poço artesiano, apresenta uma quantidade de $\mathrm{Rn}-222$ e filhos considerável quando comparada com as outras amostras. São levantadas algumas hipóteses sobre os efeitos provocados pela ingestão da água no estômago.

Palavras-chave: Detectores tipo CR-39, dose, radônio e filhos, água, poços.

\section{Radon and radon daughters' concentration in spring and wells waters from Presidente Prudente: preliminary results}

\begin{abstract}
This work presents the preliminary results about the concentration of radon and radon daughters in wells and springs water from Presidente Prudente. Six water samples were studied: three from well-water, two from springs water and one from poTabelale water. For the determination of $\alpha$-activity the samples were placed inside plastic containers where the CR-39 tracks detectors were outside the water. The track density of $\alpha$-particles were measured by using optical microscopy. The results show that one sample from well-water presented higher concentration of radon and radon daughters than the other samples.

Key words: CR-39 detectors, dose, radon and radon daughters, water, well.
\end{abstract}




\section{Introdução}

Além do solo e materiais de construção, outra fonte de Rn-222 importante em determinados tipos de ambientes é a água. Em uma investigação realizada nos EUA (DE PAULO, 1991; MONNIN, SEIDEL, 1991), verificou-se que, em um grande número de residências, a concentração de Rn-222 na cozinha superava a concentração desse elemento em outras dependências dessas residências. Outros trabalhos apresentam pesquisas realizadas em águas subterrâneas (ESPINOZA; GOLZARRI, 1991), como, por exemplo, águas de poços e cavernas, onde foram encontrados altos níveis de concentração de Rn222, como é o caso de estudos realizados na Hungria (SOMOGY; LENÁRT, 1986).

Estes resultados despertaram nosso interesse, já que na região de Presidente Prudente, Estado de São Paulo, é muito comum a utilização de águas de poços para uso doméstico. Um exemplo disto é que na cidade de Presidente Prudente se encontram instalações com várias torneiras onde a população retira a água, acreditando que estas águas, por não conterem cloro, são menos danosas à saúde. O mesmo acontece com as águas de emergências, que se encontram na margem das estradas, de onde os motoristas retiram a água que será utilizada durante a viagem ou mesmo nas suas casas. É importante lembrar que estas águas vêm de diferentes tipos de poços, e nenhum estudo foi realizado no sentido de verificar se o conteúdo de $\mathrm{Rn}-222$ nelas está dentro dos padrões internacionais ou é maior, como tem acontecido em estudos realizados em alguns países da Europa. Neste sentido, procuramos o engenheiro responsável pela análise da água da SABESP de Presidente Prudente, e verificamos que a SABESP é responsável pela análise de elementos contidos na água potável, mas não existe nenhum trabalho dirigido ao estudo das águas de emergências e poços da região. O número de poços existentes é considerável, aproximadamente 200 , daí a importância de uma pesquisa neste sentido.

\section{Métodos}

Características do Rn-222

A população é exposta a várias fontes de radiação, e entre as fontes naturais, o $\mathrm{Rn}$-222 representa aproximadamente $54 \%$ do total (MONNIN; 1991). Dentre todas as contaminações radioativas a que o homem está sujeito, a mais insalubre, do ponto de vista da população como um todo, é que diz respeito à presença de Rn-222 e filhos em ambientes de convívio humano, como explicado em De Paulo (1991).

O Rn-222 é um produto da série radioativa do urânio-238, elemento que compõe a crosta terrestre numa concentração de aproximadamente $5 p p m$. Como é um gás nobre, ele tem a capacidade de se difundir através do solo e emanar para o interior de ambientes. No entanto, outras fontes de Rn-222 são os materiais de construção e a água, o que implica que mesmo em apartamentos situados a vários metros do solo, pode haver concentrações significativas de Rn-222 (DE PAULO, 1991).

O Rn-222 pode entrar na água natural por dois processos: pelo decaimento alfa no local, do núcleo pai, o Ra-226 dissolvido na água, ou por dissolução de sólidos, gases ou ar vizinhos nas proximidades subterrâneas (ZORZETTO, 1995).

A série radioativa do urânio-238 é apresentada na Figura 1. $\alpha$ e $\beta$ representam os tipos de radioatividade envolvidos nos decaimentos. A Tabela 1 mostra algumas das características radioativas do $\mathrm{Rn}-222$ e filhos. 


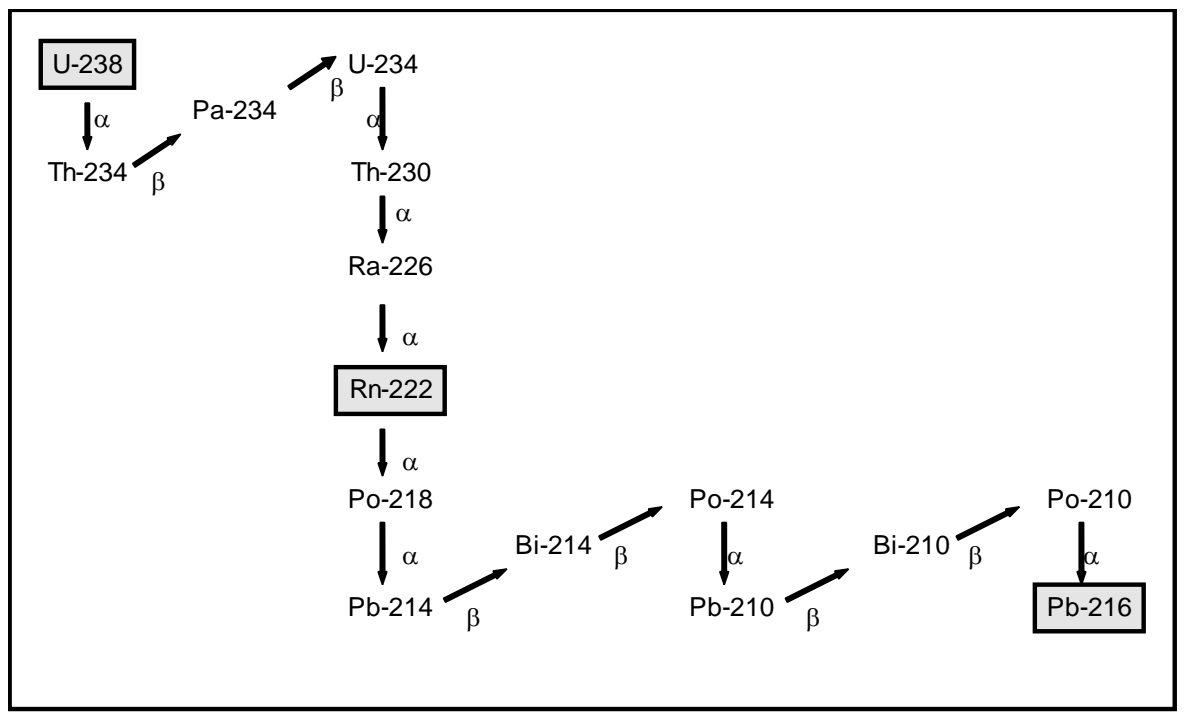

Figura 1. Série radioativa do urânio-238

Tabela 1. Características nucleares do Rn-222 e filhos.

\begin{tabular}{ccccc}
\hline Isótopos & Nome tradicional & Meia-vida & $\Lambda(\mathbf{s e g})$ & Radiação (energia) \\
\hline Rn-222 & Radônio & $3,823 \mathrm{~d}$. & $2,10 \times 10^{-6}$ & $\alpha(5,49 \mathrm{Mev})$ \\
Po-218 & Rádio A & $3,05 \mathrm{~m}$. & $3,79 \times 10^{-3}$ & $\alpha(6,00 \mathrm{Mev})$ \\
Pb-214 & Rádio B & $26,80 \mathrm{~m}$. & $4,31 \times 10^{-4}$ & $\beta, \mathrm{Y}$ \\
Bi-214 & Rádio C & $19,70 \mathrm{~m}$. & $5,86 \times 10^{-4}$ & $\beta, \mathrm{Y}$ \\
$\mathrm{Po}-214$ & Rádio C' & $164,00 \mu \mathrm{s}$ & $4,23 \times 10^{-3}$ & $\alpha(7,69 \mathrm{Mev})$ \\
$\mathrm{Pb}-210$ & Rádio D & $21 \mathrm{a}$. & $1,05 \times 10^{-9}$ & $\beta, \mathrm{Y}$ \\
$\mathrm{Bi}-210$ & Rádio E & $5,01 \mathrm{~d}$. & $1,60 \times 10^{-6}$ & $\beta$ \\
$\mathrm{Po}-210$ & Rádio F & $138,4 \mathrm{~d}$. & $5,08 \times 10^{-8}$ & $\alpha(5,30 \mathrm{Mev})$ \\
$\mathrm{Pb}-206$ & Rádio G & Estável & & \\
\hline
\end{tabular}

\section{O CR-39}

O CR-39 (allyl diglycol-carbonate- $\mathrm{C}_{12} \mathrm{H}_{18} \mathrm{O}_{7}$ ) é o detector plástico de maior eficiência, pelo menos dentre aqueles que são disponíveis comercialmente (DE PAULO, 1991). O seu desenvolvimento, como detector de traços, deu-se após 1975. Uma das grandes vantagens, além de sua eficiência, é o fato de ele ser totalmente amorfo, o que faz com que os traços sejam muito regulares. Além disto, o CR-39 é muito transparente, existindo um contraste ideal entre os traços e o próprio plástico, facilitando a observação ao microscópio óptico. Este detector é feito de um dos plásticos mais resistentes contra possíveis danos ocasionados por condições ambientais, como temperatura, umidade, ação corrosiva de 
poluentes (DE PAULO, 1991) etc. Ele encontra-se disponível comercialmente (em placas normalmente com $500 \mu \mathrm{m}$ de espessura) em apenas algumas indústrias do mundo como: American Acrilics, EUA; Homalite, EUA; Pershore Moldings, Inglaterra; Baryotrack, Japão.

O uso dos detectores plásticos, um tipo de SSNTD (Solid State Nuclear Tracks Detector), é uma das técnicas mais freqüentemente utilizadas nos dias atuais para detecção da atividade $\alpha$, devido à presença de átomos de Rn-222 e filhos em ambientes de convívio humano.

Uma "partícula pesada" (fragmentos de fissão, partículas $\alpha$, prótons etc) quando incide sobre um SSNTD, provoca um desarranjo na estrutura molecular chamado de traço latente. $\mathrm{O}$ traço tem um diâmetro da ordem de A (ângstrons) e, após um ataque químico conveniente, sua dimensão é da ordem de mícrons, e desta forma se torna observável ao microscópio óptico (FLEISCHER; PRICE; WALKER, 1975). Explicado de forma simples, o ataque químico corrói a superfície do detector e a região danificada pela partícula incidente. Em De Paulo (1991), são mencionadas as condições de ataque químico utilizadas para a revelação dos traços. Este autor obteve uma curva padrão do ataque químico para $6,25 \mathrm{~N}$ de $\mathrm{NaOH}-70^{\circ} \mathrm{C}$, onde o tempo de ataque químico escolhido foi de $400 \mathrm{~min}$.

O "background", quantidade de traços presentes no detector antes de sua utilização, é descontado das densidades de traços obtidas, já que comparado com a densidade de traços dos plásticos expostos e analisados, é desprezível.

\section{Dados geológicos e hidro-geológicos}

A região onde foram coletadas as amostras de água localiza-se em domínios da Formação Adamantina, do Grupo Bauru, do Cretáceo Superior da Bacia Sedimentar do Paraná. Do ponto de vista pedológico, de acordo com levantamento semidetalhado de solos, a área de pesquisa está situada principalmente em solos da classe Podzólico vermelho amarelo, em várias subunidades (CARVALHO et al., 1997). Trata-se de um terreno bem drenável, embora haja variação vertical na permeabilidade. A existência de horizonte "B" textual favorece o escoamento subcutâneo de água em ocasiões em que o solo se apresenta muito úmido. Uma das amostras de água (amostra A-3) foi coletada numa área que está localizada no vale do córrego da Olga, onde é mapeado o solo PEa7associação entre Podzólico vermelho escuro abrúptico epidistrófico e solos litólicos; são de drenagem relativamente lenta, com tendência a escoamento subcutâneo quando muito úmidos.

Quanto à hidrodinâmica do maciço rochoso na área pesquisada, levantamentos preliminares permitiram distinguir um padrão de comportamento do nível freático, que com corda com o padrão regional: no setor de topo aplainado do interflúvio, o limite superior da zona permanentemente saturada encontra-se a profundidades entre $10 \mathrm{~m}$ e $14 \mathrm{~m}$ da superfície, registrando-se aqui a menor oscilação sazonal; no terreno de encosta, os valores de profundidade estão entre $4 \mathrm{~m}$ e $10 \mathrm{~m}$ e no sopé da encosta, ou no fundo do vale, variam entre $2 \mathrm{~m}$ e 5 $\mathrm{m}$, com as maiores oscilações sazonais verificadas. São encontradas várias emergências de água, as quais estão provavelmente condicionadas a zonas de fraturamento locais. Zonas de ressudação ocorrem também.

No poço tubular instalado na Faculdade de Ciências e tecnologia da UNESP, é registrada a presença, crescente com a profundidade, de estratos da Formação Santo Anastácio, também do Grupo Bauru, a partir de $80 \mathrm{~m}$ de perfuração, em alternância com estratos da Formação Santo 
Anastácio. Esta unidade do grupo Bauru é constituída por arenito mais permeável que aquele correspondente à Formação Adamantina, comportando-se por isto como melhor aqüífero do que esta última.

Quanto à classificação do ponto de vista da composição química, nas duas formações há predominância de água bicarbonatada cálcica ou magnesiana (CAMPOS, 1987).

\section{Procedimento Experimental}

Foram coletadas seis amostras de água de diferentes locais segundo consta na Tabela 2. As amostras foram colocadas em recipientes plásticos que têm uma capacidade de aproximadamente $5600 \mathrm{~mL}$. Deste volume, aproximadamente 3000 $\mathrm{mL}$ foram preenchidos com água. Os recipientes foram vedados, para evitar escapamento do ar, e guardados a uma temperatura de aproximadamente $6{ }^{\circ} \mathrm{C}$, para evitar a evaporação da água que poderia se condensar na superfície do detector e interferir na medida da densidade de traços.

\section{Em cada recipiente foi colocado um} detector CR-39, colado na parte superior, mantendo-se aproximadamente $10 \mathrm{~cm}$ entre a água e o detector (DE PAULO, 1991). Após um tempo de exposição de aproximadamente 10 meses, os detectores foram retirados e submetidos a um ataque químico de $6,25 \mathrm{~N}$ de $\mathrm{NaOH}$ a $70{ }^{\circ} \mathrm{C}$ durante 400 min, segundo escrito em De Paulo (1991).
Tabela 2. Relação entre detector e amostra coletada

\begin{tabular}{ccc}
\hline $\begin{array}{c}\text { Detector } \\
\text { r }\end{array}$ & Descrição & $\begin{array}{l}\text { Tempo de } \\
\text { exposição }\end{array}$
\end{tabular}

A-1

B Detector utilizado para a medida do background Detector exposto ao ar ambiental vindo de uma amostra de água sem cloro

11 meses da UNESP

Detector exposto ao ar ambiental vindo de uma

A-2 amostra de água com cloro 10 meses da UNESP

Detector exposto ao ar ambiental vindo de uma

A-3 amostra de água de um poço artesiano localizado a 10 meses aproximadamente $15 \mathrm{Km}$ de Presidente Prudente Detector exposto ao ar ambiental vindo de uma

A-4 amostra de água coletada numa estrada vizinha a 10 meses Presidente Prudente $(8$ $\mathrm{Km})$

Detector exposto ao ar ambiental vindo de uma

A-5 amostra de água coletada numa estrada vizinha a 10 meses Presidente Prudente (40

$\mathrm{Km})$

Detector ex posto ao ar

A-6 ambiental vindo de uma 10 meses amostra de água potável

Após o ataque químico, os detectores foram analisados ao microscópio óptico para obter a densidade de traços $D$ (número de traços por 
centímetro quadrado) de partícula alfa. Esta densidade é obtida após a calibração do campo de observação e para isto é utilizada uma régua reticulada onde cada intervalo equivale a $0,01 \mathrm{~mm}$. A medida do campo de observação, em nosso caso, é de $1,3 \times 10^{-3} \mathrm{~cm}^{2}$.

Os dados são obtidos após a contagem de aproximadamente 1000 traços por cada detector e, desta forma, os erros associados às medidas são estatisticamente representativos. A densidade superficial, D, obtida para cada detector, é apresentada na Tabela 3 . O erro associado às medidas, $\varepsilon(\%)$, corresponde ao desvio padrão percentual, relativo aos valores médios.

Tabela 3. Resultados obtidos da análise dos detectores

\begin{tabular}{cccc}
\hline $\begin{array}{c}\text { Detector } \\
\mathbf{r}\end{array}$ & $\begin{array}{c}\text { № de traços / } \\
\text { № de campos }\end{array}$ & $\begin{array}{c}\text { № de traços } \\
\text { por campo }\end{array}$ & $\begin{array}{c}\mathbf{D} \pm \\
\boldsymbol{\varepsilon}(\%)\end{array}$ \\
\hline B & $1040 / 810$ & 1,28 & $\begin{array}{c}983 \pm \\
3,6\end{array}$ \\
& & & $\begin{array}{c}1251 \\
\pm 3,2\end{array}$ \\
A-1 & $1056 / 648$ & 1,63 & $\begin{array}{c} \pm 3,0 \\
5842\end{array}$ \\
& & & $\pm 3,0$ \\
A-2 & $1132 / 421$ & 2,69 & $\begin{array}{c}2265 \\
\pm 3,9\end{array}$ \\
A-3 & $2467 / 324$ & 7,61 & 2265 \\
A-4 & $1052 / 357$ & 2,95 & $\begin{array}{c}11,1 \\
\pm 21\end{array}$ \\
A-5 & $1149 / 389$ & 2,95 & \\
A-6 & $1038 / 712$ & 1,46 & $\pm 2,9$ \\
\hline
\end{tabular}

\section{Resultados e discussão}

Analisando a Tabela 3, pode-se observar que a amostra A-3 apresenta uma densidade de traços muito maior do que as outras amostras; o valor médio da densidade, é aproximadamente $300 \%$ maior do valor da densidade das outras amostras. Uma hipótese que pode ser levantada é que isto se deve possivelmente ao tipo de solo, $\mathrm{PEa} 7$, e à profundidade do poço. $\mathrm{O}$ solo onde foi coletada a amostra A-3, mesmo sendo originado da formação Adamantina, é de drenagem relativamente lenta $e$, devido à profundidade, retém mais água, fazendo com que a água fique ligada mais tempo à argila do solo, o que facilita a difusão dos átomos do Rn-222 para a água, ao contrário do que ocorre com o poço da UNESP, amostras A-1 e A. O poço da UNESP é de uma profundidade de $202 \mathrm{~m}$ e, a esta profundidade corresponde um terreno com arenito mais permeável, o que dificulta a presença de água ligada à matriz do solo. Além disto, as águas neste tipo de terreno não ficam retidas por muito tempo e são diariamente renovadas.

As amostras A-4 e A- 5 foram coletadas em emergências de água que se encontram muito distantes uma das outras, aproximadamente $50 \mathrm{~km}$. Além disto o tipo de solo é diferente. A densidade de traços nestas amostras indica uma atividade de Rn-222 e filhos pouco significativa. Uma possível explicação deste resultado é que são águas novas e constantemente renovadas. Todos os valores encontrados se encontram dentro do limite das Normas e padrão de potabilidade das águas, Portaria $n^{\circ}$. 518/GM do Ministério da Saúde.

A densidade de traços de partículas vindas do decaimento do $\mathrm{Rn}-222$ e filhos está relacionada com o cálculo da dose de radioatividade recebida, segundo descrito em De Paulo (1991). Com o intuito de avaliar se as densidades de traços mostradas na Tabela 3, correspondem a doses com ordens de grandeza comparáveis ao limite de dose permitido para a população como um todo, foi feita uma estimativa da dose-equivalente devido ao $\mathrm{Rn}$ 222 (e seus produtos de decaimento), recebida pelo aparelho digestivo, que corresponde ao órgão diretamente afetado pela ingestão de água. É 
importante destacar que este cálculo depende das características do tecido, alcance das partículas alfa nos tecidos que compõem o aparelho, e, também, o transporte dos átomos pelas células.

A estimativa da dose baseou-se no procedimento descrito em Hadler (1986, p. 82), considerando-se a quantidade de massa do aparelho digestivo, que efetivamente é exposta à radioatividade, como igual a $1 \mathrm{Kg}$. Para a relação entre a concentração do Rn-222 no ar $\left(C_{a}\right)$, contido no recipiente onde as lâminas de CR-39 foram expostas, e na água $\left(\mathrm{C}_{\mathrm{W}}\right)$, foi adotado o valor $f=1 \times 10^{-4}$, que segundo Nazaroff (1990), corresponde a um valor médio obtido através de resultados experimentais coletados por diversos pesquisadores. $F$ é denominado fator de transferência, e obedece a relação $\mathrm{C}_{\mathrm{a}}=\mathrm{f} \mathrm{C}_{\mathrm{W}}$.

Levando-se em conta as considerações acima e as densidades de traços apresentados na Tabela 3, obtivemos as doses para cada uma das amostras de água, apresentadas na Tabela 4. $\mathrm{Na}$ segunda coluna da Tabela 4 são apresentados os valores da densidade real para cada detector, ou seja, a densidade de traços obtida após descontar o background (detector B da Tabela 3) e dividir por 0,7, já que a eficiência do CR-39 para partículas $\alpha$ é de aproximadamente $70 \%$. A coluna 3 da Tabela 4 representa uma estimativa da dose recebida pelo órgão em questão, para cada amostra estudada.

Os resultados apresentados neste trabalho poderão ser confirmados com o aumento do número de amostras analisadas. Por esta razão coletamos outras amostras de água de poços da região e colocamos os detectores CR-39, nas mesmas condições descritas neste texto. Os dados obtidos serão comparados com os dados apresentados na Tabela 3 e podemos concluir sobre a importância de se realizar este tipo de pesquisa a nível regional e, também, em regiões que, como Presidente Prudente apresentam um tipo de solo que possibilita o surgimento de poços para retirada da água.

Tabela 4. Doses estimadas para cada detector

\begin{tabular}{ccc}
\hline Detector & $\mathbf{D} \pm \boldsymbol{\varepsilon}(\%)$ & Dose (mSv/ano) \\
\hline A-1 & $384 \pm 3,2$ & 1 \\
A-2 & $1546 \pm 3,0$ & 2 \\
A-3 & $6942 \pm 3,0$ & 9 \\
A-4 & $1832 \pm 3,9$ & 2 \\
A-5 & $1832 \pm 3,1$ & 2 \\
A-6 & $197 \pm 2,9$ & 0,3 \\
\hline
\end{tabular}

\section{Conclusão}

As hipóteses levantadas sobre a relação solo-água-Rn-222 são interessantes do ponto de vista da saúde pública, já que, como comentado anteriormente, estas águas são utilizadas pela população para uso doméstico. Novos dados poderão vir a confirmar nossos resultados:

As amostras, A-4 e A-5 apresentam valores onde a dose calculada está levemente acima da dose limite recomendada para a população como um todo; $1,7 \mathrm{mSv} / \mathrm{ano}$, segundo Hadler e Paulo (1986). A amostra A-3 apresenta um valor onde a dose calculada é de aproximadamente $9 \mathrm{mSv} / \mathrm{ano}$. Este valor está muito acima da dose limite recomendada. Foi obtida outra amostra do mesmo poço e está sendo analisada.

A quantidade de amostras analisadas, não nos permite, no momento, concluir se estes resultados são aplicáveis à região toda. Novas medidas e considerações sobre 0 aparelho digestivo como um todo são necessárias.

Outros trabalhos realizados pelo grupo apontam que além do tipo de solo, fatores como fraturas no sub-solo e presença de fósseis podem contribuir para a presença de ${ }^{222} \mathrm{Rn}$ nas águas. 
Agradecimentos

Os autores agradecem ao professor José Tadeu Garcia Tommaselli pela sua ajuda na interpretação de alguns resultados.

\section{Referências}

CAMPOS, H. C. N. S. Contribuição ao Estado Hidrogeoquímico do Grupo Bauru no Estado de São Paulo. 1987. 157 f. Tese (Mestrado em Geologia Geral e de Aplicação) - Instituto de Geociências da Universidade de São Paulo. São Paulo.

CARVALHO, W. A. et al. Levantamento semidetalhado dos solos da Bacia do Rio Santo Anastácio. Santo Anastácio: Faculdade de Ciências e Tecnologia - FCT, Dep. de Ciências Ambientais - FUNDACTE, 1997. (Boletim Científico ํㅜㄹ).

DE PAULO, S. R. Dosimetria ambiental de Rn222 e filhos: medida da eficiência absoluta do CR-39 levando-se em conta os efeitos do plateout e fatores ambientais. 1991. $380 \mathrm{f}$. Tese (Doutorado em Ciências) - Instituto de Física Gleb Wathagin, Universidade de Campinas. Campinas.

ESPINOZA, G.; GOLZARRI, J. I. Radon measurements of groundwater in México. Nucl. Tracks Radiat. Meas., v. 19, n. 1-4, p. 305-6, 1991. FLEISCHER, R. L.; PRICE, P. B.; WALKER, R. M. Nuclear Tracks in solids: Principles and application. Berkeley: University of California Press, 1975.

HADLER N., J. C.; PAULO, S. R. Contaminação ambiental pela radioatividade natural, In: REUNIÃO DE TRABALHOS SOBRE FÍSICA NUCLEAR NO BRASIL, 4., 1986, Caxambu, Livro de resumos, Caxambu, 1986. p. 82.
KHAN, A. J. A study of indoor radon levels in indian dwellings, influencing factors and lung cancer risks. Radiation Measurements. v. 32, p. 87-92, 2000. http://dx.doi.org/10.1016/S1350-4487(99)00275-9

MINISTÉRIO DA SAÚDE. Normas e padrão de potabilidade das águas destinadas ao consumo humano. Portaria no. 518/GM. Brasília, Brasil, março de 2004.

MONNIN M. M.; SEIDEL, J. L. Radon and geophysics: recent advances, Nucl. Tracks Radiat. Meas., v. 9, n. 1-4, p. 375-382, 1991. http://dx.doi.org/10.1016/1359-0189(91)90219-8

NAZAROFF W. W.; NERO, A. V. Radon and its decay products in indoor air. New York, USA: A Wiley Interscience Publication, 1990.

OLIVEIRA, J. et al. Determinação dos níveis de radioatividade natural presentes nas águas utilizadas para abastecimento público no Estado de São Paulo. In: Anais do Meeting on Nuclear Applications. 4, 1997. Minas Gerais. p. 18-22.

SOMOGY, G.; ENÁRT, L. Time-integrated radon measurements in spring and well waters by track technique. Nuclear Tracks, v. 12, n. 1-6, p. 731734, 1986.

ZORZETTO, N. L. Curso de anatomia humana. 6.ed. Presidente Prudente, SP: Data Júris, 1995. $224 \mathrm{f}$. 